\title{
Angiografia cerebral em cães
}

\author{
Cerebral angiography in dogs
}

\author{
Carmen Lice Buchmann de Godoy ${ }^{\mathrm{I}^{*}}$ Denise de Castro Veiga ${ }^{\mathrm{II}}$ \\ Claudete Schmidt ${ }^{\mathrm{III}}$ Luiz Carlos de Pellegrini ${ }^{\mathrm{II}}$ Stella Falkenberg Rausch ${ }^{\mathrm{III}}$
}

\section{RESUMO}

Angiografia cerebral é o método de imagem empregado para detecção de alterações dos vasos sanguíneos do cérebro, pela injeção de contraste positivo e obtenção da imagem por meio da radiografia convencional, tomografia computadorizada ou ressonância magnética. No presente trabalho, descreve-se a técnica com o emprego da radiografia convencional em razão da disponibilidade muito restrita de equipamentos de tomografia computadorizada e da inexistência de equipamentos de ressonância magnética na área da medicina veterinária, no Brasil. O objetivo foi avaliar a eficácia do meio de imagem para demonstrar o padrão angiográfico de animais sem afecção neurológica, visando a preencher a lacuna deixada pelo exame radiográfico simples, em casos de alterações de fluxo sanguíneo intracranianos. Concluiu-se que a técnica utilizada é eficaz para a obtenção de imagens nítidas dos vasos cerebrais e sua distribuição.

Palavras-chave: meio de contraste, exame radiológico, vasos cerebrais.

\section{ABSTRACT}

Cerebral angiography is an imaging technique used to detect alterations in the blood supply of the brain. A positive contrast is injected and the image obtained by conventional radiography, computerized tomography or magnetic resonance (MRI). The present research describes the technique with the use of conventional radiography due to the rare availability of tomography or MRI equipment in veterinary practice. The objective was to evaluate the efficiency of the method in demonstrating the angiographic pattern of normal animals, to serve as a complementary exam to simple $\mathrm{x}$-ray and improve the capability of detecting altered intracranial blood flow. It is concluded that the technique is capable of producing clear images of cerebral vessels and its ramifications.
Key words: contrast medium, radiological exam, cerebral vessels.

\section{INTRODUÇÃO}

A angiografia cerebral em humanos é considerada de grande valor, inclusive em pediatria, sendo realizada com relativa frequência e segurança por meio da cateterização percutânea da artéria femoral (BURGER et al., 2006). É efetuada a punção direta percutânea da artéria femoral para o sistema carotídeo, por onde se insere um cateter guiado por um fio-guia que avança pelos principais vasos do abdômen e tórax até que se encontre corretamente colocado nas artérias do pescoço. Esse procedimento é feito com acompanhamento fluoroscópico, em que a imagem dinâmica é obtida através de um feixe contínuo de radiação (MACHADO, 1992).

A angiografia cerebral continua sendo padrão ouro para o diagnóstico de lesões vasculares cerebrais extra e intracranianas, abrangendo os sistemas arterial e venoso no mesmo estudo (GURGEL, 2003). O exame é de grande valor em lesões que ocupam espaço no cérebro (PERRY \& LOWRIE, 1993), sendo considerada padrão de referência no diagnóstico de aneurismas intracranianos (KOROGI et al., 1999). Esse meio de imagem continua sendo o melhor para avaliação da angioarquitetura da malformação arteriovenosa (MAV) e dos fatores de risco que podem

'Laboratório de Diagnóstico por Imagem, Departamento de Clínica de Grandes Animais, Universidade Federal de Santa Maria (UFSM), 97105-900, Santa Maria, RS, Brasil. E-mail: cliceg@gmail.com. Autor para correspondência.

"Programa de Pós-graduação em Medicina Veterinária, UFSM, Santa Maria, RS, Brasil.

IIICurso de Medicina Veterinária, UFSM, Santa Maria, RS, Brasil. 
determinar o agravamento na evolução da mesma. Além disso, é o único meio para as análises morfoestrutural e hemodinâmica (SILVA, 2005).

A tomografia computadorizada (TC) é o primeiro exame neurorradiológico a ser realizado no caso de malformações arteriovenosas (MAVs), podendo-se observar vasos anômalos, com ou sem hemorragia associada (SILVA, 2005).

Num país de área continental como o Brasil, o reduzido número de equipamentos de TC e a ausência de RM na área veterinária são motivos para que se aplique a angiografia cerebral como meio de diagnóstico de afecções neurológicas centrais.

JAMES \& HOERLEIN (1960) aplicaram a angiografia cerebral em cães para estudos experimentais, visando a obter adequada visibilização do suprimento sanguíneo, levando em consideração que o cérebro do cão recebe sangue de duas principais fontes: as artérias carótidas internas e as artérias basilares. Naquele experimento, 20 animais foram submetidos à angiografia cerebral por meio de injeções repetidas do meio de contraste à base de amidotrizoato de meglumina na artéria carótida comum, com o clampeamento temporário da artéria carótida externa, em dose variando entre 3 e $25 \mathrm{~mL}$. Os cães foram acompanhados por um período superior a três semanas, buscando evidenciar qualquer manifestação clínica de dano cerebral. As reações descritas no período de observação de 30 minutos após o exame, em ordem de severidade, incluíram desde fracos tremores dos músculos da cabeça e do pescoço até convulsões severas acompanhadas de depressão respiratória, quando se fez necessária oxigenioterapia. Após esse período, não foram observadas alterações clínicas.

GREENE \& BRAUND (1992) utilizaram a angiografia cerebral para identificar desvio, obstrução ou aumento de vascularização do cérebro, e a desvantagem do procedimento se referia à anestesia geral, exposição cirúrgica e cateterização de uma artéria. Poderia ocorrer sepse ou embolismo, não sendo estas, no entanto, complicações frequentes.

Em um relato de estudo experimental que incluiu a interpretação de 30 angiografias e numerosas dissecações anatômicas, JUNG et al. (1975) demonstraram que existe entre cada rede da artéria carótida interna, artéria carótida externa e artéria vertebral, numerosas anastomoses e que estas funcionam permanentemente no cão, diferentemente das anastomoses no homem.

As incidências radiográficas incluem posicionamento lateral e mandibulofrontal do crânio, o que permite adequada identificação das estruturas analisadas (PERRY \& LOWRIE, 1993). A injeção do meio de contraste na artéria carótida do cão delineia o círculo arterial do cérebro (círculo de Willis) e as artérias cerebrais rostral e média. Em alguns casos, as artérias cerebral caudal, cerebelar rostral e basilar podem ser delimitadas (GREENE \& BRAUND, 1992).

O objetivo deste trabalho foi avaliar a eficácia da técnica de angiografia cerebral para demonstrar o padrão angiográfico normal de animais sem afecção neurológica, para que esse exame passe a fazer parte da rotina em pacientes suspeitos de alterações vasculares cerebrais. Esse exame deve preencher a lacuna deixada pelo exame radiográfico simples em casos de alterações de fluxo sanguíneo intracranianos, como aneurisma cerebral, tumores, hemorragias e malformações vasculares.

\section{MATERIAL E MÉTODOS}

Foram utilizados 20 cães, incluindo machos e fêmeas, sem raça definida, com massa corporal entre 14 e 17kg, em idade adulta, sem evidência clínica de alterações neurológicas, provenientes do Biotério Central da Universidade Federal de Santa Maria (UFSM). Os animais foram submetidos à avaliação clínica, por meio de ausculta cardiorespiratória, tomada de pulso, temperatura corporal, coloração de mucosas e estado de hidratação. Foi realizada coleta de amostra de sangue da veia cefálica para mensuração dos valores séricos de ureia e creatinina 24h antes do exame angiográfico, 24h após o procedimento e, novamente, sete dias após, sendo obtidos valores dentro dos parâmetros de normalidade.

Para a realização da angiografia cerebral, foi utilizado meio de contraste positivo à base de amidotrizoato sódico e amidotrizoato de meglumina ${ }^{\mathrm{a}}$. Como pré-medicação anestésica, cada animal recebeu diazepam (0,3mg kg-1), pela via intramuscular profunda. Em seguida, foi realizada ampla tricotomia da região cervical ventral e da face cranial do membro torácico esquerdo a ser abordada para acesso da artéria carótida comum esquerda e da veia cefálica esquerda, respectivamente.

$\mathrm{O}$ acesso à veia cefálica foi obtido com um cateter venoso periférico número 22, sendo essa via utilizada para a administração de fármacos e Ringer com lactato de sódio nos períodos pré e transoperatórios. A coleta de sangue foi realizada antes e após a cirurgia. A cateterização foi efetuada para a cirurgia.

No bloco cirúrgico, foi realizada a indução anestésica com a administração de tiopental sódico (12 $\mathrm{mg} \mathrm{kg}^{-1}$ ) pela via intravenosa, seguida da intubação 
orotraqueal e manutenção do plano anestésico com halotano vaporizado em oxigênio a $100 \%$, por meio de vaporizador universal.

Após os procedimentos de antissepsia, teve lugar a incisão de pele com bisturi munido de lâmina número 11, acompanhada da dissecação do tecido subcutâneo e dos planos musculares, seguida da abordagem à artéria carótida comum, localizada lateralmente à traqueia e adjacente aos nervos vago e laríngeo recorrente. A artéria foi, então, isolada com pontos de reparo em mononáilon afastados $3 \mathrm{~cm}$ entre si, sendo suspensos obliquamente e em direções opostas.

Uma tesoura de Metzenbaum foi empregada para executar uma pequena incisão na artéria. Nessa incisão, foi introduzida, em sentido cranial, uma sonda número 10 siliconizada e heparinizada com a extremidade cortada em bisel, sendo fixada ao vaso com o reparo cranial e com o caudal esta foi ligada. Não foi utilizado cateter específico para angiografia, o qual seria conduzido por fio-guia, uma vez que curto segmento introduzido na carótida foi suficiente para o procedimento. A modificação da extremidade da sonda teve como objetivo facilitar a sua introdução no interior do vaso. O reparo caudal foi ligado para evitar hemorragia.

Esse experimento foi dividido em dois grupos de 10 cães cada um. No primeiro, foi realizada a angiografia cerebral com administração do meio de contraste positivo na artéria carótida comum esquerda com seu clampeamento caudal. No segundo grupo, a variação foi em relação ao clampeamento temporário da artéria carótida esquerda cranialmente, tendo sido mantida a injeção do meio de contraste positivo na artéria carótida comum e seu clampeamento caudal. Após esses procedimentos, cada animal foi conduzido do bloco cirúrgico para o setor de radiologia veterinária.

Inicialmente, foi realizado o exame radiográfico simples, efetuando-se duas tomadas com ângulo de $90^{\circ}$ entre si. A seguir, o meio de contraste positivo foi injetado sob pressão manual, em frações de $10 \mathrm{~mL}$ para cada tomada radiográfica, chegando-se a uma dose aproximada de $20 \mathrm{~mL}$ por paciente. O tempo entre a injeção do meio de contraste e a emissão dos raios-x foi de, aproximadamente, 1,5 segundo. As incidências foram mandíbulo-frontal e lateral esquerda para os exames simples e contrastados.

Foram obtidas imagens radiográficas que tornaram evidente a área de irrigação encefálica, e foi realizada síntese, por meio da redução do espaço morto, com fio mononáilon 3-0, em padrão isolado simples. Os mesmos padrão e fio, dessa vez de calibre 4-0, foram utilizados para dermorrafia. Esses cães foram mantidos seis horas com curativo compressivo oclusivo na região cervical, em gaiola de 60x120x60 cm para sua completa recuperação e para o controle quanto a possíveis hemorragias. Como analgésico e antiinflamatório, foi utilizado cetoprofeno, solução injetável, durante três dias consecutivos, na dose de $2 \mathrm{mg} \mathrm{kg}^{-1}$ a cada 24 horas, pela via subcutânea. A realização dos curativos também ocorreu no intervalo de $24 \mathrm{~h}$, sendo nesse procedimento utilizada solução fisiológica e pomada à base de sulfato de neomicina e bacitracina. Retirados os pontos de pele em sete dias, os cães permaneceram confinados em canil e acompanhados diariamente até serem encaminhados a lares de adoção.

\section{RESULTADOS E DISCUSSÃO}

Os meios de imagem, em geral, são complementares. Mesmo que um meio possa ser conclusivo, muitas vezes são empregados dois ou três meios, como radiologia convencional, tomografia computadorizada e ressonância magnética.

SILVA (2005), avaliando MAVs, concluiu pela necessidade do estudo angiográfico cerebral para determinação dos fatores de risco, precedendo a indicação cirúrgica. No presente estudo, foi obtida imagem da vasculatura cerebral de cães com o objetivo de investigar alterações da angioarquitetura.

Como descrito por GREENE \& BRAUND (1992) em animais, bem como SILVA(2005) e BURGER et al. (2006) em humanos, neste estudo, a angiografia cerebral mostrou-se um exame passível de ser realizado com segurança, e obteve-se um resultado satisfatório mediante duas incidências radiográficas com ângulo de $90^{\circ}$ entre si. Imagens claras para determinação da área de irrigação sanguínea cerebral foram obtidas, permitindo a visibilização dos vasos preenchidos adequadamente pelo meio de contraste positivo. A figura 1 demonstra as imagens radiográficas obtidas do grupo 1 (ACC: artéria carótida comum; ACI: artéria carótida interna; ACE: artéria carótida externa; AM: artéria maxilar; ATS: artéria temporal superficial; AL: artéria lingual), e a figura 2, as imagens radiográficas do grupo 2 (AEV: artéria espinhal ventral; AV: artéria vertebral; AB: artéria basilar; ACR: artéria cerebelar rostral; ACM: artéria cerebral); ACCa: artéria comunicante caudal.

A abordagem cirúrgica da artéria carótida comum foi utilizada por não se dispor de fluoroscopia para guiar o fio-guia e o cateter, meio utilizado por MACHADO (1992). Destaca-se como relevante o pequeno calibre dos vasos em cães de menor porte para passagem do cateter, dificultando o uso deste (GREENE \& BRAUND, 1992). 

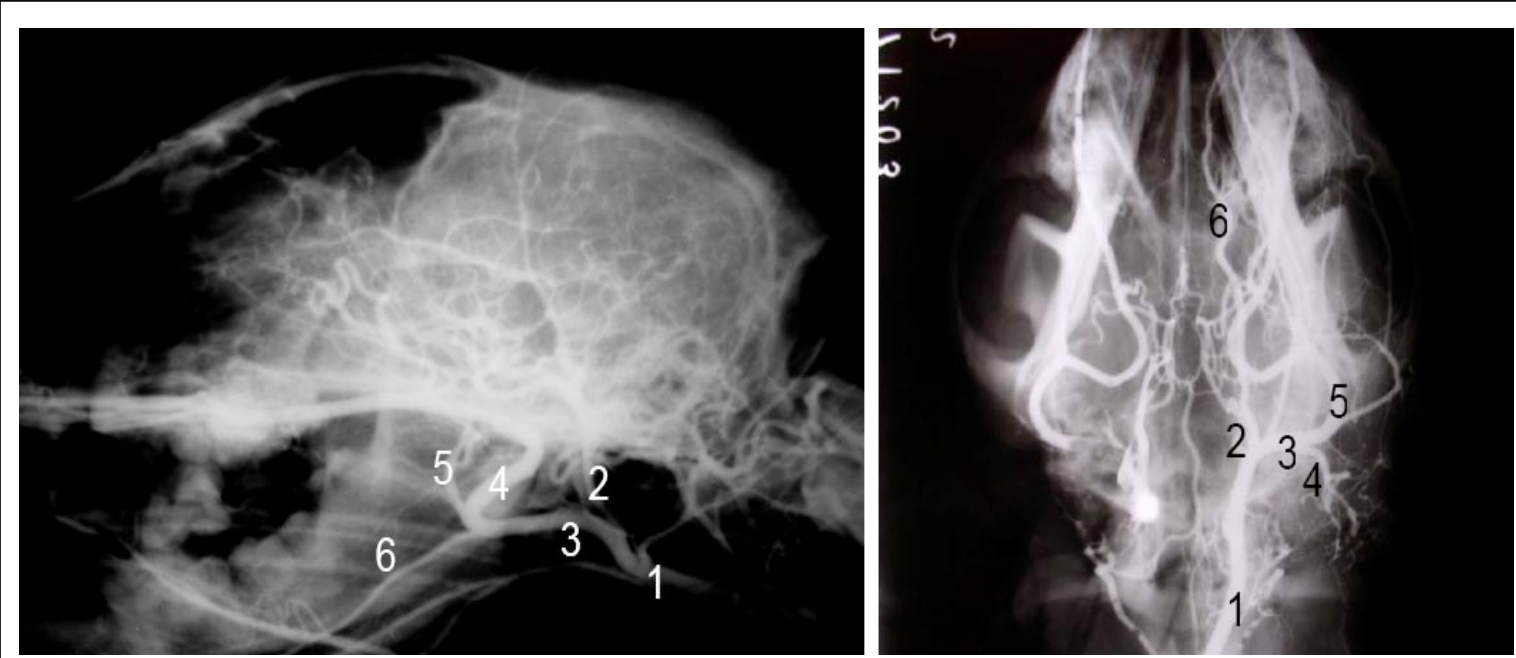

Figura 1 - Imagens radiográficas do crânio de cão nas projeções lateral esquerda e mandíbulo-frontal após administração de amidotrizoato sódico e amidotrizoato de meglumina (meio de contraste positivo), na artéria carótida comum clampeada caudalmente (grupo 1), demonstrando o preenchimento dos vasos craniais: 1-ACC: artéria carótida comum; 2-ACI: artéria carótida interna; 3-ACE: artéria carótida externa; 4-AM: artéria maxilar; 5-ATS: artéria temporal superficial; 6-AL: artéria lingual.

Em lugar do cateter periférico específico para angiografia, utilizou-se cateter uretral, considerandose que o curto segmento deste seria introduzido na artéria e levando-se em conta a grande diferença de custo entre ambos.

Enquanto JAMES \& HOERLEIN (1960) utilizaram o meio de contraste amidotrizoato de meglumina, neste estudo, foi utilizada uma associação com amidotrizoato sódico em uma dose mais elevada, e as duas incidências radiográficas foram adequadas para a visibilização dos vasos cerebrais preenchidos pelo contraste positivo. As reações heterogêneas descritas pelos autores, devido ao intervalo e a diferentes doses administradas, não se repetiram neste experimento, pois a dose foi previamente determinada, sendo as reações homogêneas e discretas. Estas consistiram em tremores da musculatura do pescoço imediatamente após a administração do contraste. Durante o período de uma

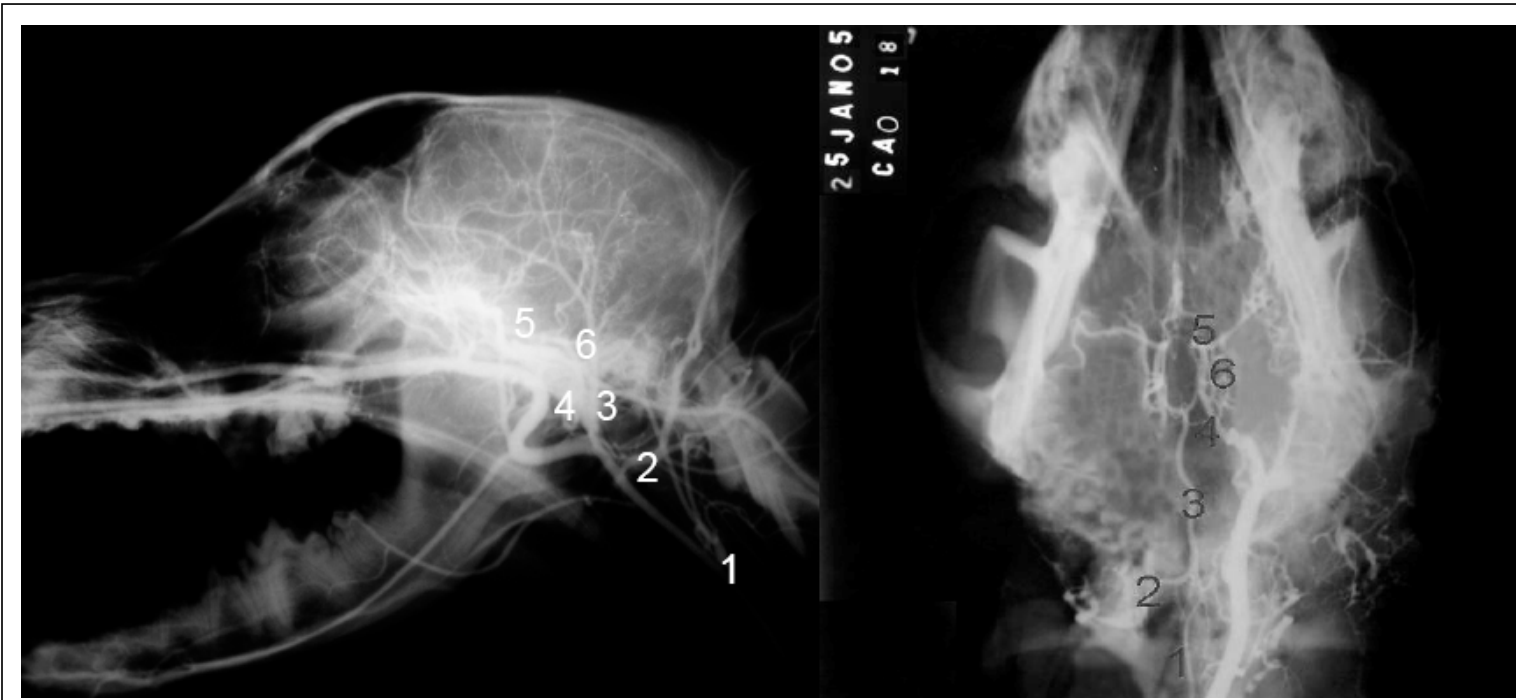

Figura 2 - Imagens radiográficas do crânio de cão nas projeções lateral esquerda e mandíbulo-frontal após administração de amidotrizoato sódico e amidotrizoato de meglumina (meio de contraste positivo), na artéria carótida comum clampeada caudalmente e artéria carótida externa clampeada cranialmente (grupo 2), demonstrando o preenchimento dos vasos craniais: 1- AEV: artéria espinhal ventral; 2-AV: artéria vertebral; 3-AB: artéria basilar; 4ACR: artéria cerebelar rostral; 5-ACM: artéria cerebral média; 6-ACCa: artéria comunicante caudal. 
semana de observação após realização do exame, nenhum dos cães apresentou alteração clínica e/ou neurológica.

\section{CONCLUSÃO}

Com base nos resultados apresentados, concluiu-se que a angiografia cerebral em cães é um exame complementar eficaz no que diz respeito à verificação da área de irrigação sanguínea cerebral de animais sob condições clínicas normais, por meio da injeção do meio de contraste positivo na artéria carótida comum. O clampeamento temporário da artéria carótida esquerda permitiu visualizar especificamente a irrigação encefálica, não havendo sobreposição de imagem dos vasos que compõem a camada mais externa.

\section{FONTES DE AQUISIÇÃO}

a - Urografina 292 - Schering do Brasil Química e Farmacêutica LTDA - Rua Cancioneiro de Évora, 255/339/383 - Santo Amaro $-\mathrm{SP}$.

\section{COMITÊ DE ÉTICA}

Este projeto foi aprovado pelo Comitê de Ética e Bem-estar Animal da Universidade Federal de Santa Maria, com parecer n⿳⺈ 40/2008.

\section{REFERÊNCIAS}

BURGER, I.M. et al. Safety of cerebral digital subtraction angiography in children. Complication rate analysis in 271 consecutive diagnostic angiograms. Baltimore: John Hopkins University, 2006. Disponível em: <http:// stroke.ahajournals.org/cgi/content/abstract/18/6/997>. Acesso em: 12 jan. 2006.
GREENE, C.E.; BRAUND, K.G. Moléstias do cérebro. In: ETTINGER, S.J. Tratado de medicina interna veterinária. São Paulo: Manole,1992. v.2, p.607-611.

GURGEL, G.A. Isquemia cerebral de origem extracraniana: diagnóstico e tratamento clínico. In: PITTA, G.B.B.; CASTRO, A.A. (Eds). Angiologia e cirurgia vascular: guia ilustrado. Maceió: UNCISAL/ECMAL \& LAVA; 2003. Disponível em: $<$ http://www.lava.med.br/livro $>$. On line. Acesso em: 08 jun. 2007.

JAMES, C.W.; HOERLEIN, B.F. Cerebral angiography in dog. Veterinary Medicine, v.21, n.2, p.45-56, 1960.

JUNG, F. et al. Angiographie des vaisseaux cervico-céphaliques du chien - Le système carotidien. Journal de Chirurgie, v.109, n.1, p.109-118, 1975.

KOROGI Y. et al. Intracranial aneurysms: detection with threedimensional CT angiography with volume rendering comparison with convencional angiographic and surgical findings. Radiology, v.211, n.2, p.497-506, 1999. Disponível em: <http://radiology.rsna.org/content/211/2.toc>. On line. Acesso em: 12 ago. 2007.

MACHADO, C.C. Criterios cubanos para el diagnostico de la muerte encefalica. Editorial Ciencias Medicas, v.101, n.4, p.121-131, 1992.

PERRY, R.L.; LOWRIE, C.T. Select contrast studies: portal venography and cerebral angiography. Veterinary Clinics of North America: Small Animal Practice, v.23, n.2, p.331343, 1993.

SILVA. M.A.B. Malformação arteriovenosa cerebral: estudo da angioarquitetura em indivíduos da região nordeste do Brasil. 2005. 42f. Dissertação (Mestrado na área de Morfologia Aplicada) - Programa de Pós-graduação em Patologia do Centro de Ciências da Saúde, Universidade Federal de Pernambuco, Recife, PE. Disponível em: $>$ biblioteca.universia.net/ficha.do? $\mathrm{id}=30069380>$. On line. Acesso em 03 mar. 2009. 